\title{
Galerkin Solutions for the Saint-Venant Torsion of Prismatic Bars with Rectangular Cross-sections
}

\author{
Charles Chinwuba Ike
}

\author{
Department of Civil Engineering, Enugu State University of Science and Technology, Enugu, 400001, Enugu State, Nigeria
}

Corresponding Author Email: ikecc2007@yahoo.com

https://doi.org/10.18280/ama_a.560103

Received: 10 November 2018

Accepted: 16 February 2019

\section{Keywords:}

galleria variational method, poison equation, Prandtl stress function, Saint Venant torsional problem

\begin{abstract}
In this work, the Saint-Venant torsion problem of prismatic bars with rectangular crosssections was presented as a boundary value problem (BVP) of the theory of elasticity. The governing partial differential equation was formulated and shown to be a Poisson equation in terms of the Prandtl stress functions. The Poisson equation governing the Saint-Venant torsion problem was expressed in variational form using Galerkin variational method. The trial function that apriori satisfies the boundary conditions was chosen as a trigonometric cosine series of infinite terms; and in terms of unknown undetermined coefficients or parameters. The unknown parameters were determined by solving the Galerkin variational integral; thus fully determining the Prandtl stress function. The shear stresses were then determined. The maximum shear stress was also obtained. The moment of the cross-section was determined and found to depend on non - dimensional torsional parameters F1(a/b). The maximum shear stress was also found to depend on dimensionless torsional parameters F2(a/b) which were determined and tabulated. It was found that the solutions obtained using the Galerkin method were mathematically closed form solutions because the exact shape functions were used to approximate the trial solution. Expressions obtained for the Prandtl stress function, shear stresses and moment of cross-section were exact and agreed with solutions in the technical literature.
\end{abstract}

\section{INTRODUCTION}

When a torque is applied to a beam with non-circular crosssection, the cross-section rotates about the longitudinal axis of the beam and simultaneously undergoes a significant distortion. The cross-section thus undergoes both twisting and warping deformations [1-8]. Such problems are formulated using theory of elasticity principles. The foundational assumptions of the formulation are the strain-displacement (kinematic) relations of infinitesimal/small deformation assumptions, the stress-strain laws, the differential equations of equilibrium and compatibility requirements. Saint-Venant formulated the problem using theory of elasticity and Prandtl solved the problem in terms of Prandtl's stress functions.

Prandtl's formulation of the Saint-Venant torsion problem leads to a Poisson type partial differential equation (PDE); which can be solved using analytical or numerical methods. Available analytical techniques include the method of separation of variables, eigenfunction expansion methods, integral transform methods and Green's function methods. The numerical methods that can be used to solve the torsion problem are the numerical methods available for solving the boundary value problems (BVP) in engineering. Some of the numerical techniques for solving BVP which are applicable to the Poisson type PDE are Galerkin's variational method, Extended Galerkin's variational method, Ritz's method, Finite Element method (FEM), Finite difference method (FDM); and Boundary element methods (BEM) [1-13].

In this work, the Saint-Venant problem of torsion of prismatic bars with rectangular cross-section will be formulated in variational form, and solved using the Galerkin variational method.

\section{Research aim and objectives}

The research aim is to use the Galerkin variational method to solve the Saint-Venant torsion problem for prismatic bars with rectangular cross-sections. The specific objectives are:

(1) to formulate the Saint-Venant torsional problem for prismatic bars with rectangular cross-sections using theory of elasticity principles and techniques

(2) to show that the formulated Saint-Venant torsional problem is governed by a partial differential equation called the Poisson equation when expressed in terms of the Airy's and Prandtl's stress functions of elasticity

(3) to express the boundary value problem (BVP) in variational form using the Galerkin variational method

(4) to solve the Galerkin variational equation for the problem and thus obtain solutions for the unknown parameters in the assumed (trial) solutions for the Airy's or Prandtl's stress function

(5) to obtain analytical expressions for the torque, shear stresses and torsional parameters

(6) to show that Galerkin's variational method can be used to obtain closed form mathematical solutions to the BVP of Saint-Venant torsion for prismatic bars with rectangular cross-sections.

\section{THEORETICAL FRAMEWORK}

The study considered an isotropic, homogeneous long bar with prismatic cross-section denoted by $R^{2}$ on the $y z$ coordinate plane. The longitudinal axis is coincident with the $x$-Cartesian coordinate axis. The bar is fixed at $x=0$. The end 
at $x=l$ is subject to a torsional moment which twists it by an angle $l \theta^{\prime}$ where $\theta^{\prime}$ is the twist rate and $l$ is the length of the bar [14-21]. Other relevant literature can be found in Roohi et al. [22] and Heydari et al. [23].

The assumptions of the formulation are as follows:

(1) the cross-sections in the $y z$ coordinate plane undergo rotation as a rigid body. For non-circular cross-sections, the cross-section will experience twisting. It is deflected in the $x-$ coordinate direction

(2) the deflection and twist rate are constant along the longitudinal axis of the bar. This renders the problem a twodimensional (2D) problem in the theory of elasticity

(3) the material of the bar is isotropic and homogeneous.

\subsection{Displacement field}

The three dimensional (3D) Cartesian components of the displacement field, following Saint-Venant hypothesis [14-21] are given by:

$$
\begin{gathered}
u(x, y, z)=\theta^{\prime} \varphi(y, z)=\beta(y, z) \\
v(x, y, z)=-\theta^{\prime} x z=-\beta x z \\
w(x, y, z)=-\theta^{\prime} x y=\beta x y
\end{gathered}
$$

where $\varphi(y, z)$ is an unknown function which is used to define the deflection and is a function of the $y$ and $z$ Cartesian coordinate variables. $u, v$, and $w$ are the components of displacement in the $x, y$ and $z$ Cartesian coordinate directions, respectively. $\beta=\theta^{\prime}$ is the twist rate.

\subsection{Strain field}

Using the strain-displacement equations for infinitesimally small deformation, the strain fields are obtained as follows:

$$
\begin{gathered}
\varepsilon_{x x}=\frac{\partial u}{\partial x}=0 \\
\varepsilon_{y y}=\frac{\partial v}{\partial y}=0 \\
\varepsilon_{z z}=\frac{\partial w}{\partial z}=0 \\
\gamma_{x y}=2 \varepsilon_{x y}=\left(\frac{\partial u}{\partial y}+\frac{\partial v}{\partial x}\right)=\beta\left(\frac{\partial \varphi}{\partial y}-z\right) \\
\gamma_{x z}=2 \varepsilon_{x z}=\frac{\partial u}{\partial z}+\frac{\partial w}{\partial x}=\beta\left(\frac{\partial \varphi}{\partial z}+y\right) \\
\gamma_{y z}=2 \varepsilon_{y z}=\frac{\partial v}{\partial z}+\frac{\partial w}{\partial y}=-\beta x+\beta x=0
\end{gathered}
$$

$\varepsilon_{x x}, \varepsilon_{y y}, \varepsilon_{z z}$ are the normal strains while $\gamma_{x y}, \gamma_{y z}$ and $\gamma_{x z}$ are the shear strains. Thus, the strain - compatibility equation becomes:

$$
\frac{\partial \gamma_{x z}}{\partial y}-\frac{\partial \gamma_{x y}}{\partial z}=\frac{\partial}{\partial y} \beta\left(\frac{\partial \varphi}{\partial z}+y\right)-\frac{\partial}{\partial z} \beta\left(\frac{\partial \varphi}{\partial y}-z\right)=\beta\left(\frac{\partial^{2} \varphi}{\partial y \partial z}+1-\frac{\partial^{2} \varphi}{\partial z \partial y}-1\right)=2 \beta
$$

Provided $\frac{\partial^{2} \varphi}{\partial y \partial z}=\frac{\partial^{2} \varphi}{\partial z \partial y}$

Eq. (11) implies that $\varphi(y, z)$ is required to be a continuous function of $y$ and $z$.

\subsection{Stress fields}

The generalized Hooke's law of linear isotropic elasticity is given generally by

$$
\tau_{i j}=\lambda \partial_{i j}\left(\varepsilon_{x x}+\varepsilon_{y y}+\varepsilon_{z z}\right)+2 G \varepsilon_{i j}=\lambda \partial_{i j} \varepsilon_{v}+2 G \varepsilon_{i j}
$$

where $\partial_{i j}=1$ for $i=j ; \partial_{i j}=0$ for $i \neq j$

$\lambda$ and $G$ are the Lamé's constants $G$ is the shear modulus. $\varepsilon_{v}$ is the volumetric strain.

$$
\varepsilon_{v}=\varepsilon_{x x}+\varepsilon_{y y}+\varepsilon_{z z}=0
$$

The stress fields are given by

$$
\begin{gathered}
\sigma_{x x}=\sigma_{y y}=\sigma_{z z}=0 \\
\tau_{x y}=G \gamma_{x y}=2 G \varepsilon_{x y}=\beta G\left(\frac{\partial \varphi}{\partial y}-z\right) \\
\tau_{x z}=G \gamma_{x z}=2 G \varepsilon_{x z}=\beta G\left(\frac{\partial \varphi}{\partial z}+y\right) \\
\tau_{y z}=G \gamma_{y z}=0
\end{gathered}
$$

where $\sigma_{x x}, \sigma_{y y}, \sigma_{z z}$ are normal stresses $\tau_{x y}, \tau_{y z}, \tau_{x z}$ are shear stresses.

\subsection{Differential equations of equilibrium}

The differential equations of equilibrium in the absence of body forces $f_{i}$ given in general by Eq. (18).

$$
\sum_{j} \partial_{j} \tau_{i j}=f_{i}=0
$$

Simplify to become Equations (19)-(21).

$$
\begin{gathered}
\frac{\partial \tau_{x y}}{\partial y}+\frac{\partial \tau_{x z}}{\partial z}=0 \\
\frac{\partial \tau_{x y}}{\partial x}=0 \\
\frac{\partial \tau_{x z}}{\partial x}=0
\end{gathered}
$$

\subsection{Prandtl's stress function $\phi(y, z)$}

Prandtl defined stress functions $\phi(y, z)$ which are functions of the $y$ and $z$ coordinate variables of the cross-section, and 
independent of $x$ such that the differential equations of equilibrium are satisfied by the non-vanishing stress components $\tau_{x y}$ and $\tau_{x z}$ as follows:

$$
\begin{gathered}
\tau_{x y}=G \beta \frac{\partial \phi}{\partial z}(y, z) \\
\tau_{x z}=-G \beta \frac{\partial \phi}{\partial y}(y, z)
\end{gathered}
$$

It is observed that for Prandtl's stress functions Eqns. (22) and (23), Eq. (19) becomes:

$$
\frac{\partial}{\partial y}\left(G \beta \frac{\partial \phi}{\partial z}\right)+\frac{\partial}{\partial z}\left(-G \beta \frac{\partial \phi}{\partial y}\right)=G \beta \frac{\partial^{2} \phi}{\partial y \partial z}-G \beta \frac{\partial^{2} \phi}{\partial z \partial y}=0
$$

if

$$
\frac{\partial^{2} \phi}{\partial y \partial z}=\frac{\partial^{2} \phi}{\partial z \partial y}
$$

Prandtl's stress functions are solutions of the differential equation of equilibrium if the functions are continuous. The strain components are given in terms of the Prandtl stress function $\phi(x, z)$ as:

$$
\begin{gathered}
\gamma_{x y}=\frac{\tau_{x y}}{G}=\beta \frac{\partial \phi}{\partial z} \\
\gamma_{x z}=\frac{\tau_{x z}}{G}=-\beta \frac{\partial \phi}{\partial y}
\end{gathered}
$$

Then the strain compatibility equation is

$$
\frac{\partial \gamma_{x z}}{\partial y}-\frac{\partial \gamma_{x y}}{\partial z}=-\beta \frac{\partial^{2} \phi}{\partial y^{2}}-\beta \frac{\partial^{2} \phi}{\partial z^{2}}=-\beta\left(\frac{\partial^{2} \phi}{\partial y^{2}}+\frac{\partial^{2} \phi}{\partial z^{2}}\right)
$$

From Eq. (10), Eq. (25) can be expressed as:

$$
-\beta\left(\frac{\partial^{2} \phi}{\partial y^{2}}+\frac{\partial^{2} \phi}{\partial z^{2}}\right)=2 \beta
$$

Simplifying,

$$
\Delta \phi=\frac{\partial^{2} \phi(y, z)}{\partial y^{2}}+\frac{\partial^{2} \phi(y, z)}{\partial z^{2}}=\nabla^{2} \phi(y, z)=-2
$$

where

$$
\Delta=\nabla^{2}=\frac{\partial^{2}}{\partial y^{2}}+\frac{\partial^{2}}{\partial z^{2}}
$$

$\Delta$ or $\nabla^{2}$ is the Laplace differential operator.

\subsection{Boundary condition}

The boundary condition for the Prandtl stress function for a cross-sectional profile with no holes is

$$
\phi(y, z)=0
$$

on the boundary $\Gamma$.

\subsection{Torque, section moment and shear stresses}

The torque or torsional moment, $M$ is computed as the double integral over the cross-section

$$
M=\iint_{R^{2}}\left(-\tau_{x y} z+\tau_{x z} y\right) d y d z
$$

where $R^{2}$ is the cross-section of the bar.

$$
\begin{array}{r}
M=\iint_{R^{2}}\left(-G \beta \frac{\partial \phi}{\partial z} z-G \beta \frac{\partial \phi}{\partial y} y\right) d y d z \\
M=-G \beta \iint_{R^{2}}\left(\frac{\partial \phi}{\partial z} z+\frac{\partial \phi}{\partial y} y\right) d y d z
\end{array}
$$

Using the method of integration by parts,

$$
\begin{gathered}
\iint_{R^{2}} \frac{\partial \phi}{\partial z} z d y d z=\int_{\Gamma} \phi z n_{z} d s-\iint_{R^{2}} \phi(y, z) d y d z=-\iint_{R^{2}} \phi(y, z) d y d z \\
\iint_{R^{2}} \frac{\partial \phi}{\partial y} y d y d z=-\iint_{R^{2}} \phi(y, z) d y d z \\
M=2 G \beta \iint_{R^{2}} \phi(y, z) d y d z=G \beta J
\end{gathered}
$$

where

$$
J=2 \iint_{R^{2}} \phi(y, z) d y d z
$$

$J$ is the moment of the cross-section, or torsional constant. The modulus of the shear stress is

$$
|T|=\left(\tau_{x y}^{2}+\tau_{x z}^{2}\right)^{1 / 2}
$$

\section{METHODOLOGY}

For a rectangular cross-section on the $y z$ Cartesian coordinate plane defined by

$$
-\frac{a}{2} \leq \mathrm{y} \leq \frac{a}{2} ;-\frac{b}{2} \leq \mathrm{z} \leq \frac{b}{2}
$$


where $a \geq b>0$ the Prandtl stress function that satisfies the boundary condition Eq. (32) is assumed in terms of the unknown parameters $C_{m n}$ as the infinite series:

$$
\phi(y, z)=\sum_{m}^{\infty} \sum_{n}^{\infty} C_{m n} \cos \frac{m \pi y}{a} \cos \frac{n \pi z}{b}
$$

$m=1,3,5,7,9, \ldots ; n=1,3,5,7,9, \ldots$

Since

$$
\phi\left(y= \pm \frac{a}{2}, z\right)=\phi\left(y, z= \pm \frac{b}{2}\right)=0
$$

The Galerkin variational integral becomes:

$$
\int_{-b / 2}^{b / 2} \int_{-a / 2}^{a / 2}\left(\nabla^{2} \phi+2\right) \cos \frac{m^{\prime} \pi y}{a} \cos \frac{n^{\prime} \pi z}{b} d y d z=0
$$

Expanding,

$$
\int_{-b / 2}^{b / 2} \int_{-a / 2}^{a / 2}\left\{\left(\frac{\partial^{2}}{\partial y^{2}}+\frac{\partial^{2}}{\partial z^{2}}\right)\left(\sum_{m}^{\infty} \sum_{n}^{\infty} C_{m n} \cos \frac{m \pi y}{a} \cos \frac{n \pi z}{b}\right)+2\right\} \cos \frac{m^{\prime} \pi y}{a} \cos \frac{n^{\prime} \pi z}{b} d y d z=0
$$

\section{RESULTS}

The Galerkin variational integral is

$$
\begin{gathered}
\int_{-b / 2}^{b / 2} \int_{-a / 2}^{a / 2} \sum_{m}^{\infty} \sum_{n}^{\infty}\left(\frac{\partial^{2}}{\partial y^{2}}+\frac{\partial^{2}}{\partial z^{2}}\right)\left(c_{m n} \cos \frac{m \pi y}{a} \cos \frac{n \pi z}{b}\right) \\
\cos \frac{m^{\prime} \pi y}{a} \cos \frac{n^{\prime} \pi z}{b} d y d z \\
=\int_{-b / 2}^{b / 2} \int_{-a / 2}^{a / 2}-2 \cos \frac{m^{\prime} \pi y}{a} \cos \frac{n^{\prime} \pi z}{b} d y d z \\
\sum_{m}^{\infty} \sum_{n}^{\infty}-C_{m n} \int_{-b / 2-a / 2}^{b / 2} \int_{b / 2}^{a / 2}\left(\left(\frac{m \pi}{a}\right)^{2}+\left(\frac{n \pi}{b}\right)^{2}\right) \cos \frac{m \pi y}{a} \cos \frac{n \pi z}{b} \cos \frac{m^{\prime} \pi y}{a} \cos \frac{n^{\prime} \pi z}{b} d y d z \\
=\int_{-b / 2}^{a} \int_{-a / 2}-2 \cos \frac{n^{\prime} \pi z}{b} \cos \frac{m^{\prime} \pi y}{a} d y d z
\end{gathered}
$$

-2 is expanded in Fourier cosine series as:

$$
-2=\sum_{m}^{\infty} \sum_{n}^{\infty} a_{m n} \cos \frac{m \pi y}{a} \cos \frac{n \pi z}{b}
$$

where

$$
\begin{aligned}
a_{m n} & =\frac{4}{a b} \int_{-b / 2}^{b / 2} \int_{-a / 2}^{a / 2}(-2) \cos \frac{m \pi y}{a} \cos \frac{n \pi z}{b} d y d z \\
a_{m n} & =\frac{4}{a b} \cdot-2 \cdot \frac{2 a}{m \pi}(-1)^{\frac{m-1}{2}} \frac{2 b}{n \pi}(-1)^{\frac{n-1}{2}}
\end{aligned}
$$

$$
a_{m n}=\frac{(-2) 4^{2}}{m n \pi^{2}}(-1)^{\frac{m+n-2}{2}}=\frac{(-2) 4^{2}(-1)^{\frac{m+n}{2}-1}}{m n \pi^{2}}
$$

So,

$$
-2=\sum_{m}^{\infty} \sum_{n}^{\infty}(-1)^{\frac{m+n}{2}-1} \frac{(-2) 4^{2}}{m n \pi^{2}} \cos \frac{m \pi y}{a} \cos \frac{n \pi z}{b}
$$

So,

$$
\begin{aligned}
& \sum_{m}^{\infty} \sum_{n}^{\infty}-C_{m n} \int_{-b / 2-a / 2}^{b / 2} \int_{a / 2}^{a}\left(\left(\frac{m \pi}{a}\right)^{2}+\left(\frac{n \pi}{b}\right)^{2}\right) \cos \frac{m \pi y}{a} \cos \frac{n \pi z}{b} \cos \frac{m^{\prime} \pi y}{a} \cos \frac{n^{\prime} \pi z}{b} d y d z \\
& =\sum_{m}^{\infty} \sum_{n}^{\infty} \int_{-b / 2}^{b / 2} \int_{-a / 2}^{a / 2} \frac{(-1)^{\frac{m+n}{2}-1}(-2)(4)^{2}}{m n \pi^{2}} \cos \frac{m \pi y}{a} \cos \frac{n \pi z}{b} \cos \frac{m^{\prime} \pi y}{a} \cos \frac{n^{\prime} \pi z}{b} d y d z \\
& C_{m n}=\frac{(2) 4^{2}(-1)^{\frac{m+n-2}{2}}}{m n \pi^{2}}\left(\left(\frac{m \pi}{a}\right)^{2}+\left(\frac{n \pi}{b}\right)^{2}\right)^{-1}=\frac{2^{5}(-1)^{\frac{m+n}{2}-1}}{m n \pi^{2}}\left(\frac{1}{\pi^{2}\left(\left(\frac{m}{a}\right)^{2}+\left(\frac{n}{b}\right)^{2}\right)}\right) \\
& =\frac{2^{5}(-1)^{\frac{m+n}{2}-1}}{m n \pi^{4}}\left(\frac{1}{\left(\frac{b^{2} m^{2}+n^{2} a^{2}}{a^{2} b^{2}}\right)}\right) \\
& C_{m n}=\frac{2^{5}(-1)^{\frac{m+n}{2}-1} a^{2} b^{2}}{\pi^{4}} \frac{1}{m n\left(m^{2} b^{2}+n^{2} a^{2}\right)}
\end{aligned}
$$

The Prandtl stress function is then:

$$
\phi(y, z)=\frac{2^{5} a^{2} b^{2}}{\pi^{4}} \sum_{m}^{\infty} \sum_{n}^{\infty} \frac{(-1)^{\frac{m+n}{2}-1} \cos \frac{m \pi y}{a} \cos \frac{n \pi z}{b}}{m n\left(m^{2} b^{2}+n^{2} a^{2}\right)}
$$

$\phi(y, z)$ is obtained as a trigonometric cosine series of infinite terms. The series is a convergent series since

$$
\begin{aligned}
& \left|C_{m n} \cos \frac{m \pi y}{a} \cos \frac{m \pi z}{b}\right| \leq\left|C_{m n}\right| \leq \operatorname{constant}\left(\frac{1}{m^{2} n^{2}}\right) \quad \text { and } \\
& \sum_{m}^{\infty} \sum_{n}^{\infty} \frac{1}{m^{2} n^{2}}=\left(\sum_{m}^{\infty} \frac{1}{m^{2}}\right)\left(\sum_{n}^{\infty} \frac{1}{n^{2}}\right)
\end{aligned}
$$

\subsection{Moment of the cross-section (torsional constant, $j$ )}

From Eq. (39),

$$
\begin{gathered}
J=2 \int_{-b / 2}^{b / 2} \int_{-a / 2}^{a / 2} \frac{2^{5} a^{2} b^{2}}{\pi^{4}} \sum_{m}^{\infty} \sum_{n}^{\infty} \frac{(-1)^{\frac{m+n}{2}-1} \cos \frac{m \pi y}{a} \cos \frac{n \pi z}{b}}{m n\left(m^{2} b^{2}+n^{2} a^{2}\right)} d y d z \\
J=\sum_{m}^{\infty} \sum_{n}^{\infty} \frac{2^{6} a^{2} b^{2}}{\pi^{4}} \frac{(-1)^{\frac{m+n}{2}-1}}{m n\left(m^{2} b^{2}+n^{2} a^{2}\right)} \int_{-b / 2}^{b / 2} \int_{-a / 2}^{a / 2} \cos \frac{m \pi y}{a} \cos \frac{n \pi z}{b} d y d z \\
J=\frac{2^{8} a^{3} b^{3}}{\pi^{6}} \sum_{m}^{\infty} \sum_{n}^{\infty} \frac{1}{m^{2} n^{2}\left(m^{2} b^{2}+n^{2} a^{2}\right)}
\end{gathered}
$$

Let 


$$
\frac{a}{b}=r
$$

or

$$
a=b r
$$

then,

$$
\begin{aligned}
& J=\frac{2^{8} b^{6} r^{3}}{\pi^{6}} \sum_{m}^{\infty} \sum_{n}^{\infty} \frac{1}{m^{2} n^{2}\left(m^{2} b^{2}+n^{2} b^{2} r^{2}\right)} \\
& J=\frac{2^{8} b^{6} r^{3}}{\pi^{6}} \sum_{m}^{\infty} \sum_{n}^{\infty} \frac{1}{m^{2} n^{2} b^{2}\left(m^{2}+n^{2} r^{2}\right)} \\
& J=\frac{2^{8} b^{4} r^{3}}{\pi^{6}} \sum_{m}^{\infty} \sum_{n}^{\infty} \frac{1}{m^{2} n^{2}\left(m^{2}+n^{2} r^{2}\right)}
\end{aligned}
$$

Alternatively,

$$
J=\frac{2^{8} a^{6}}{r^{3} \pi^{6}} \sum_{m}^{\infty} \sum_{n}^{\infty} \frac{1}{m^{2} n^{2}\left(\frac{m^{2} a^{2}}{r^{2}}+n^{2} a^{2}\right)}
$$$$
J=\frac{2^{8} a^{6}}{r^{3} \pi^{6}} \sum_{m}^{\infty} \sum_{n}^{\infty} \frac{1}{m^{2} n^{2}\left(\frac{m^{2} a^{2}+n^{2} a^{2} r^{2}}{r^{2}}\right)}
$$$$
J=\frac{2^{8} a^{6}}{r^{3} \pi^{6}} \sum_{m}^{\infty} \sum_{n}^{\infty} \frac{r^{2}}{m^{2} n^{2} a^{2}\left(m^{2}+n^{2} r^{2}\right)}
$$$$
J=\frac{2^{8} a^{6}}{r \pi^{6}} \sum_{m}^{\infty} \sum_{n}^{\infty} \frac{1}{m^{2} n^{2} a^{2}\left(m^{2}+n^{2} r^{2}\right)}
$$

$$
J=\frac{2^{8} a^{4}}{r \pi^{6}} \sum_{m}^{\infty} \sum_{n}^{\infty} \frac{1}{m^{2} n^{2}\left(m^{2}+n^{2} r^{2}\right)}
$$

or

$$
\begin{gathered}
J=\frac{2^{8} a^{3} b^{3}}{\pi^{6}} \sum_{m}^{\infty} \sum_{n}^{\infty} \frac{1}{b^{2} m^{2} n^{2}\left(\frac{m^{2} b^{2}+n^{2} a^{2}}{b^{2}}\right)} \\
J=\frac{2^{8} a^{3} b}{\pi^{6}} \sum_{m}^{\infty} \sum_{n}^{\infty} \frac{1}{m^{2} n^{2}\left(m^{2}+\frac{n^{2} a^{2}}{b^{2}}\right)} \\
J=\frac{2^{8}}{\pi^{6}} \sum_{m}^{\infty} \sum_{n}^{\infty} \frac{(a / b)^{2}}{m^{2} n^{2}\left(m^{2}+\frac{n^{2} a^{2}}{b^{2}}\right)} \cdot a b^{3}
\end{gathered}
$$

where

$$
J=F_{1}\left(\frac{a}{b}\right) a b^{3}
$$

$$
F_{1}\left(\frac{a}{b}\right)=\frac{2^{8}}{\pi^{6}} \sum_{m}^{\infty} \sum_{n}^{\infty} \frac{(a / b)^{2}}{m^{2} n^{2}\left(m^{2}+\frac{n^{2} a^{2}}{b^{2}}\right)}
$$

\subsection{Shear stress tensors}

The shear stresses are found from the Prandtl stress function as:

$$
\begin{gathered}
\tau_{x y}(y, z)=G \beta \frac{\partial \phi}{\partial z}(y, z)=G \beta \frac{\beta^{5} a^{2} b^{2}}{\pi^{4}} \sum_{m}^{\infty} \sum_{n}^{\infty} \frac{(-1)^{\frac{m+n}{2}-1}}{m n\left(m^{2} b^{2}+n^{2} a^{2}\right)} \cos \frac{m \pi y}{a} \frac{\partial}{\partial z} \cos \frac{n \pi z}{b} \\
\tau_{x z}(y, z)=-G \beta \frac{\partial \phi}{\partial y}(y, z)=-G \beta \frac{2^{5} a^{2} b^{2}}{\pi^{4}} \sum_{m}^{\infty} \sum_{n}^{\infty} \frac{(-1)^{\frac{m+n}{2}-1}}{m n\left(m^{2} b^{2}+n^{2} a^{2}\right)} \cos \frac{n \pi z}{b} \frac{\partial}{\partial y}\left(\cos \frac{m \pi y}{a}\right)
\end{gathered}
$$

$$
\begin{gathered}
\tau_{x y}(y, z)=\frac{\beta G 2^{5} a^{2} b}{\pi^{3}} \sum_{m}^{\infty} \sum_{n}^{\infty} \frac{(-1)^{\frac{m+n}{2}}}{m\left(m^{2} b^{2}+n^{2} a^{2}\right)} \cos \frac{m \pi y}{a} \sin \frac{n \pi z}{b} \\
m=1,3,5,7,9, \ldots ; \mathrm{n}=1,3,5,7,9, \ldots \\
\tau_{x z}(y, z)=-G \beta \frac{2^{5} a b^{2}}{\pi^{3}} \sum_{m}^{\infty} \sum_{n}^{\infty} \frac{(-1)^{\frac{m+n}{2}}}{n\left(m^{2} b^{2}+n^{2} a^{2}\right)} \sin \frac{m \pi y}{a} \cos \frac{n \pi z}{b} \\
m=1,3,5,7,9, \ldots ; \mathrm{n}=1,3,5,7,9, \ldots
\end{gathered}
$$

The maximum shear stress $\tau_{\max }$ is found as:

$$
\begin{gathered}
\tau_{\max }=\frac{G \beta 2^{5} a^{2}}{\pi^{3} b} \sum_{m}^{\infty} \sum_{n}^{\infty}\left\{\frac{(-1)^{\frac{m-1}{2}}}{m\left(m^{2}+n^{2} \frac{a^{2}}{b^{2}}\right)}\right\} \\
\tau_{\max }=G \beta a b^{2} F_{2}\left(\frac{a}{b}\right)
\end{gathered}
$$

where

$$
F_{2}\left(\frac{a}{b}\right)=F_{1}\left(\frac{a}{b}\right) \frac{\pi^{3}}{2^{5}\left(\frac{a}{b}\right)^{2}}\left\{\sum_{m}^{\infty} \sum_{n}^{\infty} \frac{(-1)^{\frac{m-1}{2}}}{m\left(m^{2}+\frac{n^{2} a^{2}}{b^{2}}\right)}\right\}
$$

The non-dimensional torsional parameters $F_{1}\left(\frac{a}{b}\right)$ and $F_{2}\left(\frac{a}{b}\right)$ for the Saint Venant torsion of prismatic bars with rectangular cross-sections are tabulated as Tables 1 and 2 for various values of the ratio $a / b$ for the present study and $\bar{F}_{1}, \bar{F}_{2}$ are for results from Jan Francu et al. [3]. 
Table 1. Variation of torsional parameter $F_{1}$ with $a / b$

\begin{tabular}{ccc}
\hline $\mathbf{r}=\mathbf{a} / \mathbf{b}$ & $\boldsymbol{F}_{\mathbf{1}}(\boldsymbol{a} / \boldsymbol{b})$ & $\overline{\boldsymbol{F}_{\mathbf{1}}}(\boldsymbol{a} / \boldsymbol{b})[\mathbf{3}]$ \\
\hline 1 & 0.1406 & 0.141 \\
1.2 & 0.1661 & \\
1.5 & 0.1958 & 0.196 \\
2 & 0.2287 & 0.229 \\
2.5 & 0.2494 & \\
3 & 0.2633 & 0.263 \\
4 & 0.2808 & 0.281 \\
5 & 0.2913 & 0.291 \\
6 & 0.298 & 0.298 \\
8 & 0.307 & 0.307 \\
10 & 0.3123 & 0.312 \\
$\infty$ & $1 / 3$ & $1 / 3$ \\
\hline
\end{tabular}

Table 2. Variation of torsional parameter $F_{2}$ for with $a / b$ for Saint Venant torsion of bar with rectangular section

\begin{tabular}{ccc}
\hline $\mathbf{r}=\mathbf{a} / \mathbf{b}$ & $\boldsymbol{F}_{\mathbf{2}}(\boldsymbol{a} / \boldsymbol{b})$ & $\overline{\boldsymbol{F}_{\mathbf{2}}}(\boldsymbol{a} / \boldsymbol{b})[3]$ \\
\hline 1 & 0.208 & 0.208 \\
1.5 & 0.231 & 0.231 \\
2 & 0.246 & 0.246 \\
3 & 0.267 & 0.267 \\
4 & 0.282 & 0.282 \\
5 & 0.292 & 0.292 \\
6 & 0.299 & 0.299 \\
8 & 0.307 & 0.307 \\
10 & 0.313 & 0.313 \\
$\infty$ & $1 / 3$ & $1 / 3$ \\
\hline
\end{tabular}

The deflection function $\varphi(y, z)$ is obtained from solving the following equations obtained from Eqns. (7), (8), (26) and (27):

$$
\frac{\partial \varphi}{\partial y}-z=\frac{\partial \phi}{\partial z}
$$

and

$$
\frac{\partial \varphi}{\partial z}+y=-\frac{\partial \phi}{\partial y}
$$

Thus,

$$
\frac{\partial \varphi}{\partial y}=\frac{\partial \phi}{\partial z}+z
$$

or,

$$
\frac{\partial \varphi}{\partial z}=-\frac{\partial \phi}{\partial y}-y
$$

By integration of Eq. (83) we obtain Eq. (85) as $\varphi(y, z)$

$$
\varphi(y, z)=\frac{2^{5} a^{3} b}{\pi^{4}} \sum_{m}^{\infty} \sum_{n}^{\infty} \frac{(-1)^{\frac{m+n}{2}} \sin \frac{m \pi y}{a} \sin \frac{n \pi y}{b}}{m^{2}\left(m^{2} b^{2}+n^{2} a^{2}\right)}+y z
$$

\subsection{Numerical problem}

A numerical problem to illustrate the validity of the results obtained in this study considers the calculation of torsional constant $J$, given by the expressions in Eqns. (72) and (73) where Eq. (73) is presented as Table 1 in terms of $a / b$. The torsional contant is important in torsion problems since it determines the torsional rigidity $D_{t}$ as follows:

$$
D_{t}=G J
$$

We compare our results with results from Jan Francu et al. [3] who presented a Navier series solution of the torsion problem leading to results that are identical with the results from the present study which employed the Galerkin method.

Numerical solutions are presented for various values of $a$

\begin{tabular}{|c|c|c|c|c|c|c|}
\hline \multicolumn{2}{|c|}{ Cross-section } & \multirow[t]{2}{*}{$a / b$} & \multirow[t]{2}{*}{$F_{1}$} & \multirow{2}{*}{$\begin{array}{c}J=a b^{3} F_{1} \\
\begin{array}{c}\text { Present } \\
\text { study }\end{array} \\
\left(\mathrm{cm}^{4}\right)\end{array}$} & \multirow[t]{2}{*}{$F_{1}$} & \multirow{2}{*}{$\begin{array}{c}\mathbf{J} \\
=\underset{a}{a} \boldsymbol{b}^{3} \overline{\boldsymbol{F}_{1}} \\
\begin{array}{c}\text { Jan } \\
\text { Francu }\end{array} \\
\text { et al [3] }\end{array}$} \\
\hline$a(\mathrm{~cm})$ & $\begin{array}{c}b \\
(\mathrm{~cm})\end{array}$ & & & & & \\
\hline 2 & 2 & 1 & 0.1406 & 2.2496 & 0.141 & 2.256 \\
\hline 4 & 2 & 2 & 0.2287 & 7.3184 & 0.229 & 7.328 \\
\hline 6 & 2 & 3 & 0.2633 & 12.6384 & 0.263 & 12.624 \\
\hline 8 & 2 & 4 & 0.2808 & 17.9712 & 0.281 & 17.984 \\
\hline 10 & 2 & 5 & 0.2913 & 23.304 & 0.292 & 23.28 \\
\hline 12 & 2 & 6 & 0.298 & 28.604 & 0.298 & 28.604 \\
\hline 16 & 2 & 8 & 0.307 & 39.296 & 0.307 & 39.296 \\
\hline 20 & 2 & 10 & 0.3123 & 49.968 & 0.312 & 49.92 \\
\hline$\infty$ & 2 & $\infty$ & $1 / 3$ & $\infty$ & $1 / 3$ & $\infty$ \\
\hline
\end{tabular}
and $b$ as follows and compared with results from Jan Francu et al. [3].

Table 3. Results for torsional stiffness for various crosssections, and comparison with results from Jan Francu et al.

[3]

\section{DISCUSSION}

This work has successfully presented the Saint-Venant torsion problem of prismatic bars with rectangular crosssection as a boundary value problem (BVP) of the theory of elasticity using Prandtl's stress function $\phi(y, z)$. The resulting BVP was observed to be a Poisson type partial differential equation in terms of the Prandtl's stress functions. The basis (shape) functions that satisfies the boundary conditions given in terms of trigonometric (cosine) functions; and the assumed (trial) Prandtl stress function used was given as Eq. (45) - a double trigonometric cosine series of infinite terms.

The Galerkin variational formulation of the Saint-Venant torsion equation was obtained as Eq. (45). The unknown parameters of the Galerkin formulation was obtained by solving the Galerkin variational statement of the Poisson equation as Eq. (54). The Prandtl stress function was thus completely determined as Eq. (55), which was found to be a rapidly convergent double trigonometric cosine series with infinite terms.

The moment of the cross-section was obtained in terms of the ratio of the cross-sectional dimensions $(a / b)$ as Eqns. (71), and (72) where Eq. (72) is expressed in terms of the nondimensional torsion parameter, $F_{1}(a / b) F_{1}(a / b)$ is found to depend on the ratio $(a / b)$ as Eq. (73). Values of $F_{1}(a / b)$ for various values of $a / b$ were calculated and shown in Table 1.

The non-vanishing shear stress fields were found as Eqns. (76) and (77). The maximum shear stress was obtained as Eq. 
(78) and presented in terms of the dimensionless torsion parameter $F_{2}(a / b)$ as Eq. (79). The dimensionless torsion parameter $F_{2}(a / b)$ was calculated for various values of $(a / b)$ and presented as Table 2 .

The numerical results obtained for the torsional constant $J$ for various values of the cross-sectional dimensions were identical with the results obtained by Jan Francu et al. [3] who used Navier series method.

\section{CONCLUSIONS}

The conclusions of this study are as follows:

(1) the Galerkin variational method has been successfully used to present the Poisson equation for the SaintVenant torsion of prismatic bars with rectangular cross-section in variational form.

(2) the Galerkin solutions yielded mathematically closed form and exact solutions to the Saint-Venant torsion problem of prismatic bars with rectangular cross-section.

(3) Exact solutions were obtained because the shape functions used were exact shape functions which apriori satisfied all the boundary conditions.

(4) the exact solutions obtained for the Prandtl stress function, the shear stresses and moment of the cross-section were convergent series with infinite terms.

(5) the solutions obtained were closely similar to the solutions obtained by Jan Francu et al. [3] who used Navier series method.

\section{REFERENCES}

[1] Abdelkader, K., Toufik, Z., Mohamed, B.J. (2015). Torsional stress in non-circular cross-sections by the finite element method. Advances in Mechanical Engineering, $\quad 7(5)$ : 1-20. https://doi.org/10.1177/1687814015581979

[2] Torsion Introduction. https//www.public.iastate.edu/ m.424/Torsion\%20intr o\%20Plus. pdf.

[3] Francu, J., Novackova, P., Janicek, P. (2012). Torsion of a Non - circular bar. Engineering Mechanics, 19(1): 4560.

[4] Barber, J.R. (2002). Elasticity. Springer, Netherlands. http://doi.org/10.1007/0-306-48395-5

[5] Sadd, M.H. (2014). Elasticity: Theory, Applications and Numerics. 3rd Edition. Academic Press, USA.

[6] Srinath, L.S. (2009). Advanced Mechanics of Solids. Third Edition. Tata McGraw Hill Publishing Company Limited, New Delhi.

[7] Shalpari, S., Hematiyan, M.R. (2013). Closed form solutions for torsion analysis of structural beams considering web-flange junction fillets. Journal of Theoretical and Applied Mechanics, Warsaw, 51(2): 393-407.

[8] Ecsedi, I., Baksa, A. (2010). Prandtl's formulation for the Saint-Venant's torsion of homogeneous piezoelectric beams. International Journal of Solids and Structures, 47(22): https://doi.org/10.1016/j.ijsolstr.2010.07.007

[9] Epele, L.N., Fanchiotti, H., Garcia Canal, C.A. (2012). General solution of laplace and poisson equations in a multiply connected circular domain: Applications to torsion. SIAM Journal on Applied Mathematics, Society for Industrial and Applied Mathematics (SIAM), 72(3): 919-934. https://doi.org/10.1137/110852231

[10] Abbasi Nasser, M. (2006). Review of FEM solution for the torsion problem of a rectangular cross-section. https/12000.org/my_courses/UCI_COURSES/CREDIT .../index.htm. Nov. 27 2006. Accessed on 10 Aug. 2018.

[11] Selvadurai, A.P.S. (2000). Partial Differential Equation in Mechanics 2. The Biharmonic Equation, Poisson's Equation. Springer, New York.

[12] Liu, C.S. (2006). New methods for elastic torsion of bar with arbitrary shape of cross-section. Proceedings of Symposium on Advances of Mechanics in Honour of President Robert R. Hwang, May 12, 2006. Keelung, Taiwan, pp. 1-7.

[13] Timoshenko, S.P., Goodier, J.M. (1969). Theory of Elasticity. 3rd Edition. McGraw Hill, New York.

[14] Little, R.W. (1973). Elasticity. Prentice-Hill, New Jersey.

[15] Ockendon, J.R., Howison, S.D., Lacey, A.A., Movchan, A.B. (2003). Applied Partial Differential Equations Revised Edition. Oxford University Press, Oxford, Great Britain.

[16] Eslami, M.R. (2014). Finite elements methods in mechanics solid mechanics and its applications. Springer International Publishing Switzerland. https://doi.org/10.1007/978-3-319-08037-6

[17] Teimoni, H., Faal, R.T., Das, R. (2016). Saint-Venant torsion analysis of bars with rectangular cross-section and effective coating layers. Applied Mathematics and Mechanics, 37(2): 237-253. https://doi.org/10.1007/s10483-016-2028-8

[18] Jog, C.S., Mokashi, I.S. (2014). A finite element method for the Saint-Venant torsion and bending problems for prismatic beams. Computers and Structures, Pergamon Press, New York, USA, 135: 62-72. https://doi.org/10.1016/j.compstruc.2014.01.010

[19] Karihaloo, B.L., Xiao, Q. (2016). St Venant torsion and bending of prismatic composite shafts. Proceedings Indian National Science Academy, 82(2): 183-200.

[20] Ecsedi, I. (2009). Some analytical solutions for SaintVenant torsion of non-homogeneous cylindrical bars. European Journal of Mechanics -A/Solids. Elsevier 2009, 28(5): 985. https://doi.org/10.1016/j.euromechsol.2009.03.010

[21] Romano, G., Barretta, A., Barretta, R. (2012). On torsion and shear of Saint-Venant beams. European Journal of Mechanics A/Solids, 35(2012): 47-60. https://doi.org/10.1016/j.euromechsol.2012.01.007

[22] Roohi, R., Heydari, M.H., Aslami, M., Mahmoudi, M.R. (2018). A comprehensive numerical study of space-time fractional bioheat equation using fractional - order legendre functions. The European Physical Journal Plus, 133(10): 412. https://doi.org/10.1140/epjp/i2018-12204$\mathrm{x}$

[23] Heydari, M.H., Mahmoudi, M.R., Shakibia, A., Avazzadeh, Z. (2018). Chebyshev cardinal wavelets and their application in solving nonlinear stokchastic differential equations with fractional Brownian motion. Communications in Nonlinear Science and Numerical Simulation, $\quad$ Elsevier, 64: 98-121. https://doi.org/10.1016/j.cnsns.2018.04.018 


\section{NOMENCLATURE}

$x, y, z$

$u(x, y, z)$

$v(x, y, z)$

$w(x, y, z)$

$y$, and $z$

$\theta^{\prime}=\beta$

$\varphi(y, z)$

$\varepsilon_{x x}, \varepsilon_{y y}, \varepsilon_{z z}$

$\gamma_{x y}, \gamma_{x z}, \gamma_{y z}$

$\lambda$

G

$D_{t}$

$\varepsilon_{v}$

$\partial_{i j}$

$\tau_{i j}$

$\varepsilon_{i j}$

$\sigma_{x x}, \sigma_{y y}, \sigma_{z z}$

$\tau_{x y}, \tau_{x z}, \tau_{y z}$

$\phi(y, z)$

$R^{2}$

$M$

$\Gamma$

$J$

$m, n, m^{\prime}, n^{\prime}$

$a, b$

$C_{m n}$
Cartesian coordinates in three dimensions

Displacement field components in the $x$,

Cartesian coordinate directions

twist rate

unknown function related to deflection and used to define the deflection

normal strains

shear strains

Lamé's content

shear modulus or modulus of rigidity

torsional rigidity

volumetric strain

Kronecker's delta

stress using indicial notation

strain using indicial notation

normal stresses

shear stresses

Prandtl's stress function

cross-section of the bar

torque, torsional moment

boundary of the cross-section

moment of the cross-section, St Venant

torsional constant

integers

in-plane dimensions (length and width)

unknown parameter of the Prandtl's

stress function $a_{m n} \quad$ cosine series parameter

$r$

$F_{1}(a / b)$

$\left.F_{2}(a / b)\right\}$

aspect ratio

$\bar{F}_{1}(a / b)$

$\left.\bar{F}_{2}(a / b)\right\}$

2D

3D

BEM

BVP

PDE

FEM

FDM

dimensionless torsion parameters

dimensionless torsion parameters

obtained by Jan Francu et al. [3] using

Navier series method

two dimensional

three dimensional

boundary element method

boundary value problem

partial differential equation

finite element method

finite difference method

\section{MATHEMATICAL SYMBOLS}

$\sum \quad$ summation

$\sum \Sigma \quad$ double summation

$\int \quad$ integration (integral)

$\iint \quad$ double integration (double integral)

$\frac{\partial}{\partial x} \quad$ partial derivative with respect to $x$

$\frac{\partial}{\partial y} \quad$ partial derivative with respect to $y$

$\frac{\partial^{2}}{\partial x \partial y} \quad$ mixed partial derivative

$\Delta=\nabla^{2} \quad$ Laplacian 